\title{
Energía nuclear, ¿qué está en juego: beneficio económico o bienestar socioambiental?
}

\section{Nuclear energy, what is at stake: economic benefit or socio-environmental well-being?}

\author{
Aleida Azamar Alonso \\ Yolanda M. García Beltrán*•
}

\section{Resumen}

El objetivo de este artículo es estudiar la relevancia de la energía nuclear en México tanto para la economía como para el Estado y la población.Además, analizar la oposición del movimiento social Madres Veracruzanas que se originó por la implementación de la Central Nuclear Laguna Verde (CNLV) en el estado deVeracruz. La metodología empleada fue la de estudio de caso para valorar de forma integral si la CNLV representa una alternativa energética viable para el país. Como resultado se verificó que la CNLv no es factible a nivel económico y ambiental debido a los altos costos de producción y a los riesgos ecológicos; por otro lado, a nivel social hay desconfianza y no existe alguna propuesta por parte del Estado. Se concluye que la CNLV no representa una opción viable de largo plazo, pues provoca conflictos sociales, degradación ambiental y costos económicos elevados.

Palabras clave: energía nuclear, Laguna Verde, Madres Veracruzanas, costos, resistencia.

\begin{abstract}
The objective of this article is to study the relevance of nuclear energy in Mexico for the economy, as well as for the State and population. In addition, to analyze the opposition of the "Madres Veracruzanas" social movement that originated from the implementation of the LagunaVerde nuclear power plant (CNLV) in the state of Veracruz. The methodology used was that of a case study to fully assess whether the CNLV represents a viable energy alternative for the country. As a result, it was verified that the CNLV is not feasible at an economic and environmental level due to high production costs and ecological risks; on the other hand, at the social level there is mistrust and there is no proposal on the part of the State. It is concluded that the CNLV does not represent a viable long-term option, since it causes social conflicts, environmental degradation and high economic costs.
\end{abstract}

Keywords: nuclear energy, Laguna Verde, Madres Veracruzanas, risks, costs, resistance.

\footnotetext{
-Doctora en Economía Internacional y Desarrollo, por la Universidad Complutense de Madrid. Profesora-investigadora en el Departamento de Producción Económica, Universidad Autónoma Metropolitana, Unidad Xochimilco. gioconda 15@gmail.com Orcid:0000-0002-79I5-76II.

- Maestra en Ciencias Sociales por la Universidad Autónoma del estado de Morelos. Estudiante del Doctorado de Ciencias Sociales, Universidad Autónoma Metropolitana, Unidad Xochimilco. ymgbl988@ gmail.com Orcid: 0000-0001-9258-0783.

Fecha de recepción: 5 de mayo de 2020. Fecha de aceptación: 15 de marzo de 2021.
} 
Introducción

El presente trabajo se realizó con la premisa de evaluar multidimensionalmente a la Central Nuclear Laguna Verde (CNLV), la cual presta un servicio de provisión eléctrica en México y es una de las varias opciones energéticas que existen en el país (adicionales a los hidrocarburos), esto con la intención de verificar si es una alternativa solvente que permita dejar atrás a los combustibles fósiles más contaminantes y costosos.

Con el fin de realizar un análisis integral mucho más eficiente, se optó por emplear parcialmente la metodología de estudio de caso, tratando de lograr con ello un mayor alcance en las implicaciones sociales que ha tenido la CNLV para la comunidad local al considerar las observaciones del principal colectivo que se opone a este proyecto, por lo que se realizaron entrevistas semiestructuradas ${ }^{1}$ en diversos periodos temporales a las integrantes de la agrupación Madres Veracruzanas para conocer y comprender los efectos de dicho rechazo, así como para valorar sus argumentaciones. Además, se planteó una propuesta teórica basada en la conflictividad social y la tensión de territorialidades como un marco de análisis sobre la imposición de proyectos energéticos con un alto nivel de riesgo ante la desaprobación colectiva.

Finalmente, se realizó una revisión cuantitativa de varios indicadores económicos sobre las condiciones necesarias para la creación de una planta nuclear, su posterior desmantelamiento, así como los costos de mantenimiento necesarios para la producción durante toda su vida útil, considerando también cuáles son las necesidades económicas que se

I. Se partió de preguntas específicas previamente formuladas, pero no se siguió un esquema rígido durante la conversación con las integrantes de Madres Veracruzanas, pues surgieron otras interrogantes a lo largo de las pláticas originadas por las mismas respuestas de las entrevistadas. 
deben cubrir para aumentar la extensión de la CNLV como se ha estado planteando en México. Asimismo, se revisó la eficiencia productiva de la planta frente a otras opciones "sustentables", de manera que se pudiera medir adecuadamente si todos estos elementos, sumados a la parte social, convierten a la CNLV en una opción viable para México.

La revisión de datos estadísticos se obtuvo de informes, anuarios y documentos de la Secretaría de Energía; BP plc; Bloomberg; International Energy Agency; World Nuclear Association, y de la Plataforma México, Clima y Energía.

Este trabajo se desarrolla en tres apartados. En el primero se realiza una breve revisión de algunas categorías analíticas empleadas para comprender la naturaleza de los conflictos socioambientales. En este sentido, esta sección sirve para caracterizar y entender por qué existe rechazo social a los proyectos de aprovechamiento energético y cuáles son los elementos que identifican parte de las motivaciones para la resistencia del colectivo analizado en este texto. En la segunda sección se estudian los antecedentes de la Central Nucleoeléctrica de Laguna Verde y su viabilidad económica; asimismo, se realizan comparaciones con otras opciones tecnológicas más baratas o eficientes y se analiza si existen elementos que justifiquen el interés de mantener operativa esta planta o incrementar su tamaño. En el tercer apartado se efectúa una revisión del movimiento Madres Veracruzanas que se desarrolla en el estado de Veracruz en México, ya que dicho colectivo se opone a la narrativa del Estado por imponer una planta de energía nucleoeléctrica. Además, se recupera parte de los testimonios emanados de esta resistencia y se mencionan las interacciones de las mujeres que protagonizan, organizan y llevan a cabo esta lucha. Finalmente, se presentan las conclusiones. 


\section{Conflictos socioambientales} y marcos para su comprensión

De acuerdo con Fernanda Paz, los conflictos socioambientales nacen por la amenaza a la existencia de proyectos productivos intensivos con alto impacto ambiental y social al que se enfrentan las comunidades más vulnerables. La consideración de Paz (2014) se vincula con el carácter fundamental economicista de cualquier proyecto de Estado basado en la explotación ambiental, pues el interés final es la reproducción del capital y no la protección de las múltiples interdependencias en la relación sociedad-naturaleza que se defienden y se expresan mediante las resistencias colectivas.

Y es que, si bien es la normatividad administrativa sobre la naturaleza lo que permite la existencia de estas condiciones de riesgo, estas reglas de trabajo exponen el vínculo entre lo público y lo privado - Estado-empresa-, con el fin de garantizar la reproducción del capital de la que se nutre la administración pública. Si bien la relación de las grandes corporaciones con el Gobierno mexicano se ha transformado a lo largo del tiempo y cada sexenio guarda sus propias particularidades en este sentido, en general es perceptible que los intereses privados y gubernamentales están guiados por una lógica depredadora en la que se busca el control de los bienes naturales que permiten la reproducción del capital, y no el bienestar social.

Por lo anterior, las características del proyecto que se analiza en este trabajo son parte de un proceso de dominación del territorio, privatización de un espacio común y de amenaza constante a la vida, en el que se ven relacionados intereses institucionales con fines de intensificación productiva, pues es importante considerar que si bien en el caso particular de Laguna Verde nos encontramos ante una iniciativa gubernamental donde la Comisión Federal de Electricidad (CFE) es la principal responsable, los daños 
ecológicos y sociales están presentes de la misma forma que en cualquier otro megaproyecto financiado por particulares.

Específicamente, el proyecto de Laguna Verde fue concebido durante una época cuando el Estado tenía un papel central como figura de mando, pero su inauguración coincide con la apertura que el gobierno de Carlos Salinas de Gortari manifestó ante el sector privado. De esta manera, aunque Laguna Verde nace como producto de una necesidad por generar energía en el país y como un plan proveniente de la institucionalidad gubernamental mexicana, poco a poco ha dado cabida a compañías privadas que la proveen de ensambles de combustible nuclear y servicios asociados de ingeniería (Celis, 2021). Por otro lado, vale la pena recordar que el actual Gobierno encabezado por Andrés Manuel López Obrador se ha planteado la ampliación de Laguna Verde con cuatro nuevos reactores que serán concesionados a privados (Rodríguez, 2019).

Sin embargo, más allá de la dicotomía público-privado, se trata de entender que cada gobierno ha implementado sus propias políticas en materia energética. Así, por ejemplo, durante el sexenio del ex presidente Enrique Peña Nieto fue evidente el interés de privilegiar a los proyectos de energía renovable encomendados a compañías trasnacionales. Ahora bien, López Obrador, en su calidad de mandatario, oficializó en marzo de 2021 la nueva Ley de la Industria Eléctrica, que busca justamente retirar los privilegios otorgados por su antecesor al sector privado y conducir a un rescate de la cFE y de Pemex. No obstante, a la par la llamada cuarta transformación impulsa otros megaproyectos como la construcción de la Refinería Dos Bocas, el Aeropuerto de Santa Lucía y el Tren Maya, cuyo bien común y financiamiento público no excluye los impactos socioculturales ni el carácter depredador de los mismos.

Asimismo, es menester señalar que la problemática del abuso en el uso de los territorios es compleja y radica en 
varias cuestiones que de manera general se analizan en este artículo, mismas que generan oposición social debido a los elementos que se presentan en el siguiente esquema:

Diagrama 1. Caracterización de los efectos negativos que provocan resistencia social a diversos proyectos

a)

- Daños ambientales irreversibles en el metabolismo social local.

- Debilitamiento del tejido comunitario a raiz de la destrucción identitaria por la apropiación y destrucción territorial.

- Aumento de la marginación local debido a la inequidad productiva del modelo basado en la explotación ambiental.

d)

- Privatización y cercamiento de los bienes comunes naturales.

- Acciones violentas e ilegales por parte de algunas empresas o de las e) instituciones gubernamentales contra los detractores de los proyectos.

Fuente: elaboración propia.

Estas cinco características exteriorizan parte de la naturaleza dominante, así como el carácter anti-vida que comparten gran parte de los proyectos energéticos o extractivos en el país. No es de sorprender que generen sentimientos de rechazo por quienes se ven afectados, pues justo como lo menciona Paz (2014), se trata de procesos que ponen en riesgo a comunidades enteras. No obstante, también debe señalarse la importancia de reconocer que existe una parte de la población que está a favor de la realización de estos proyectos, lo que generalmente se da por las condiciones económicamente adversas que el mismo sistema capitalista provoca, influyendo en su flexibilización sobre la destruc- 
ción y apropiación territorial. La suma de posturas locales (a favor y en contra de los proyectos energéticos) genera otro tipo de conflictos socioambientales enmarcados en la debilidad del tejido comunitario y en la negación misma del metabolismo social local.

La identificación de elementos que provocan la confrontación de las poblaciones con la explotación territorial se deriva de que estas actividades afectan la individualidad y el bienestar colectivo de los pueblos cercanos a las mismas, lo que conlleva al surgimiento de movimientos de resistencia, tema que se puede abordar a partir de la perspectiva de la acción colectiva de Touraine (2006), desde la conceptualización de tensión de territorialidades de Porto Gonçalves (2001), así como también con la narrativa del ecofeminismo. ${ }^{2}$

Para Touraine la acción colectiva es el eje de los movimientos sociales, el cual puede definirse como la capacidad de organización y los elementos que le dan sentido; es decir, lo que hace que exista un reconocimiento entre los agentes como sujetos con un fin común. En esta lógica, para el autor existen tres principios fundamentales que dan pie al desarrollo de los movimientos sociales: a) el de la identidad, que engloba la autopercepción de los actores y las relaciones sociales que hacen que nazca una identificación entre ellos; b) el de oposición, que "solamente puede surgir si el actor se siente confrontado con una fuerza social en donde la disputa es por las orientaciones generales de la vida social" (Kuri, 2016: 197), y c) el de totalidad, que corresponde al contexto y las condiciones previas que permiten que se desarrolle "una visión societal diferente a las dominantes” (Kuri, 2016: 197).

2. "El ecofeminismo como pensamiento y movimiento social se refiere [...] a la conexión ideológica entre la explotación de la naturaleza y la explotación de las mujeres dentro del sistema jerárquico-patriarcal” (Gebara, 2000: 18). Si bien el ecofeminismo tiene una gran y muy amplia riqueza conceptual, en este trabajo no se profundiza en las múltiples formas de interpretación de ésta, por lo que se simplifica con la conceptualización de Gebara. 
Lo que Touraine llama principio de totalidad va íntimamente ligado con lo que Porto Gonçalves (2001) denomina tensión de territorialidades, pues ambas visiones refieren a un choque de intereses, ideologías o cosmovisiones. Para Touraine (2006) tienen gran importancia el enjeu (aquello por lo que se lucha) y la identidad. Es así como la totalidad está principalmente dada por una lucha de clases.

Mientras que para Gonçalves (2001), el punto medular es la manera en la que esas diferencias se materializan en el espacio en forma de confrontaciones por el territorio a través de dos sistemas: el ambientalismo y el neoliberalismo, y el choque principal se da entre una territorialidad oficial, instituida por el Estado bajo la premisa desarrollo-progreso, y otras formas de relacionarse con el territorio que éste ha excluido.

En la narrativa de Touraine y de Gonçalves existe una heterogeneidad en la composición colectiva que crea el entramado de accionar social y político de confrontación con las conductas coercitivas que ejercen los aparatos en el poder para llevar a cabo sus intereses productivos. No obstante, aunque estos análisis se basan en la pluralidad de existencia y diversificación representativa (de clase, género, naturaleza, entre otras), lo cierto es que en esta condición se invisibiliza el actuar de diversos grupos. Por ejemplo, las mujeres, entre muchos otros grupos subalternos, suelen pasar inadvertidas en la integración de propuestas y de prácticas realizadas para la defensa del territorio. Es importante establecer esta aclaración, pues mediante el estudio de posiciones específicas se brinda un matiz de análisis mucho más amplio que el que está construido desde lo general.

En esta perspectiva aparece la propuesta del ecofeminismo anti-extractivista en el Abya Yala ${ }^{3}$ que se trata en

3. Vocablo de origen indígena del pueblo conocido como los guna, quienes habitan la región de Panamá y Colombia. Algunos representantes indígenas de la zona sur de América Latina se refieren así al continente en su conjunto, como una 
sí de una recuperación de cuestiones fundamentales sobre la lucha por mantener la identidad local ante la imposición de proyectos de explotación y transformación ecoterritorial y socioambiental, así como del importante papel que tienen las mujeres en la creación del tejido comunitario y en la formación de múltiples relaciones de vida que conllevan a la creación de movimientos de resistencia en torno a la defensa del territorio.

La particularidad que conecta esta visión con la de Touraine y Gonçalves es que desde el ecofeminismo se reconoce la presencia de un proceso colonial de explotación humana (principalmente femenina) y natural para garantizar la existencia del modelo de producción capitalista, que además integra y compone parte de las capas institucionales que le dan forma al metabolismo social humano. En la praxis del ecofeminismo se vislumbra el rompimiento profundo con la lógica de producción y de coerción del modelo económico, trasladándose hacia un proyecto comunitario que fomenta la estructura identitaria.

De esta manera, en una gran cantidad de casos los proyectos dan lugar a tensiones territoriales, conflictos socioambientales e intervención política y social de diferentes actores y que, en algunos casos, conducen al surgimiento de resistencias, de acción colectiva y de movimientos sociales. Un ejemplo de esto se presenta en la CNLV en Veracruz, en donde parte de la población se ha manifestado en contra por diversos factores, sobre todo sociales, pero en este análisis también se observa que existen pocos beneficios económicos y prácticos a futuro, por lo que estos elementos pueden generar mayor desconfianza por parte de la sociedad hacia este tipo de actividades productivas. En el siguiente apartado se mencionan algunas de las cuestiones que sirven para valorar la viabilidad de esta planta nuclear.

forma de rechazo a la imposición de títulos establecidos durante la conquista y colonización de la región. 
Central Nucleoeléctrica de Laguna

Verde: antecedentes y características

En la región sureste de México se encuentra el estado de Veracruz, el cual cuenta con una extensión territorial de $71,699 \mathrm{~km}^{2}$, lo que corresponde a $3.58 \%$ de la superficie nacional. La población de la entidad es de 7.6 millones de habitantes. A pesar de no ser uno de los territorios más grandes en dimensión en el país, en los últimos años en dicha entidad han tenido lugar múltiples conflictos de corte socioambiental que giran en torno al rechazo a la minería, a las hidroeléctricas y al fracking principalmente, siendo una de las entidades que mayor número de reportes de este tipo concentra (González, 2019). Sin embargo, uno de los primeros casos y, posiblemente, el más icónico, es el surgimiento de la oposición a la Central Nucleoeléctrica de Laguna Verde (CNLV), la única de este tipo en la nación y que actualmente produce $4.02 \%$ de la energía generada en México (Secretaría de Energía, 2018a).

El desarrollo de la planta nuclear estuvo motivado principalmente por la búsqueda de nuevas tecnologías durante la década de los cincuenta del siglo pasado e inicialmente fue vista como una opción viable debido a la gran cantidad de energía que podría producir; también a que se creía que no generaría gases de efecto invernadero (GEI).

La intención era mejorar la eficiencia productiva por el uso de nuevos materiales, ciencias y recursos que los conflictos bélicos habían provocado a nivel internacional. Después de la Segunda Guerra Mundial, la energía nuclear fue uno de los motivos de competencia entre Estados Unidos (EUA) y la ex Unión Soviética (URSS) debido a sus evidentes y siniestros usos militares. Es hasta mediados de la década de los cincuenta que comienza a operar en la URSs la primera central nuclear para proveer energía eléctrica. 
Para ese entonces México se robusteció notablemente en el tema energético, tanto por el crecimiento industrial como por el aumento poblacional, y en 1956 se creó la Comisión Nacional de Energía Nuclear (CNEN) con el fin de investigar todos los procesos referentes a la energía nuclear a nivel nacional, con excepción de la generación de energía eléctrica. Esta última responsabilidad se le delegó a la CFE, por lo que en 1966 se empiezan a buscar sitios para instalar una central nucleoeléctrica que ayudara a proveer de energía eléctrica al país. Es hasta 1976 que comienza a construirse CNLV en el municipio de Alto Lucero de Gutiérrez Barrios, a la orilla de la costa del Golfo de México y muy cerca del poblado Palma Sola, Veracruz. Dicha construcción tardaría 24 años hasta que, finalmente, el 29 de julio de 1990 inició operaciones.

Cuando la CNLV comenzó a operar, la energía nuclear mantenía un crecimiento constante en la matriz energética ${ }^{4}$ del mundo. En la década de los noventa casi alcanzaba 10\% de toda la energía eléctrica generada y se esperaba que fuera creciendo, hasta existían análisis de esos años que apostaban porque estas plantas proveyeran entre 35 y $50 \%$ hacia el año 2020, pero desde la primera década de este siglo comenzó a decaer la cantidad de energía que proveen dichas plantas, debido al abaratamiento masivo de otras fuentes de energía renovables. Actualmente la energía nuclear aporta poco menos de $7 \%$ del total de energía eléctrica en el mundo (véase imagen 1 ).

4. Es la representación gráfica de las múltiples formas o fuentes de energía primaria que se emplean en un determinado espacio geográfico. 
Imagen 1. Consumo energético mundial

por tipo de combustible (1985-2018, en \%)

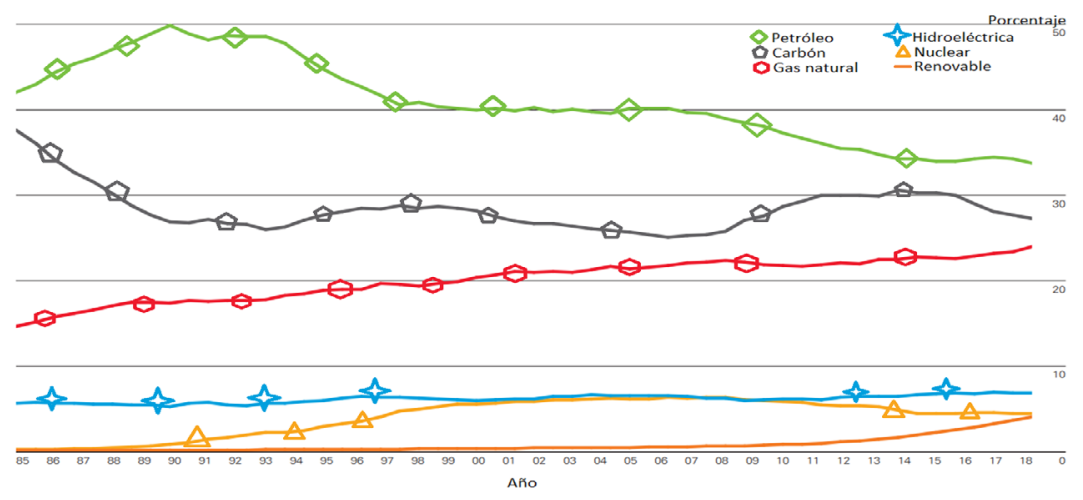

Fuente: tomada de bp Statistical Review of World Energy (2019: 11).

Para el caso de México, la energía nuclear generó 4.02\% del total de electricidad en el año 2018 (Secretaría de Energía, 2018a) y de acuerdo con el New Energy Outlook 2019, este productor tenderá a mantener constante su participación hasta el año 2035, cuando comenzará a menguar por el notable incremento de otras opciones de tipo renovables como la eólica y solar, las cuales posiblemente dominarán la matriz energética del país a partir de esa década. Este escenario concuerda con otros parecidos que han pronosticado importantes analistas en el tema en este año respecto a la evolución de la producción eléctrica en un futuro muy próximo.

A pesar de lo comentado nuestro país va a contracorriente, ya que la actual Secretaría de Energía encabezada por Rocío Nahle ha sugerido la ampliación de la CNLV:

Nosotros tenemos sólo el 4.3 por ciento (de producción nucleoeléctrica) y deberíamos contemplar, hacer un estudio, para hacer un crecimiento [...] creo que en México no hemos comunicado bien este tema. 
Decimos "nucleoeléctrica" y nos da miedo por los dos accidentes que han pasado en Rusia y Japón (El Financiero, 2019).

Lo anterior implica que en México la dinámica de explotación y apropiación territorial no ha cambiado y se encuentra todavía cercana a procesos productivos extractivistas de corte local con interés de fortalecer dinámicas capitalistas que en la actualidad ya son poco funcionales para naciones más desarrolladas, tanto por la amenaza social que representan como por los costos económicos. De este modo, por ejemplo, Bélgica que cuenta con siete reactores nucleares que en 2019 llegaron a producir casi $50 \%$ de la energía (El Periódico de la Energía, 2020) contempla cerrarlos definitivamente para 2025 (Albisu, 2020); lo mismo ocurre con otros países de la Unión Europea que consideran esta tecnología "poco segura"; por ejemplo Alemania, después del accidente nuclear en Fukushima, Japón, en 2011, se propuso abandonar este tipo de energía para 2022 y en su lugar ha apostado por la eólica y la solar (La Vanguardia, 2020). Un caso similar encontramos en Suiza, que cerró su primera central nuclear en diciembre de 2019 (La Vanguardia, 2019).

El hecho de que cada vez más países europeos estén tomando esta decisión debido a los costos ambientales y el riesgo que representan las centrales nucleares, sugiere que las productoras energéticas que participan en este sector se han convertido en depredadoras, ya que para evitar su desaparición en los mercados internacionales promueven sus dinámicas a través de cabildeos administrativos en naciones periféricas. Lo anterior concuerda con la evaluación del tema nuclear realizado por la International Energy Agency (2018) para el periodo 2017-2030, en el que se establece que este tipo de plantas productoras tendrán un comportamiento negativo en su crecimiento dentro de las economías más desarrolladas, mientras que en las subdesarrolladas afianzarán su participación (véase imagen 2). 
Imagen 2. Cambios en la demanda energética nuclear por tipo de economía (En mtep*, 2017-2030)

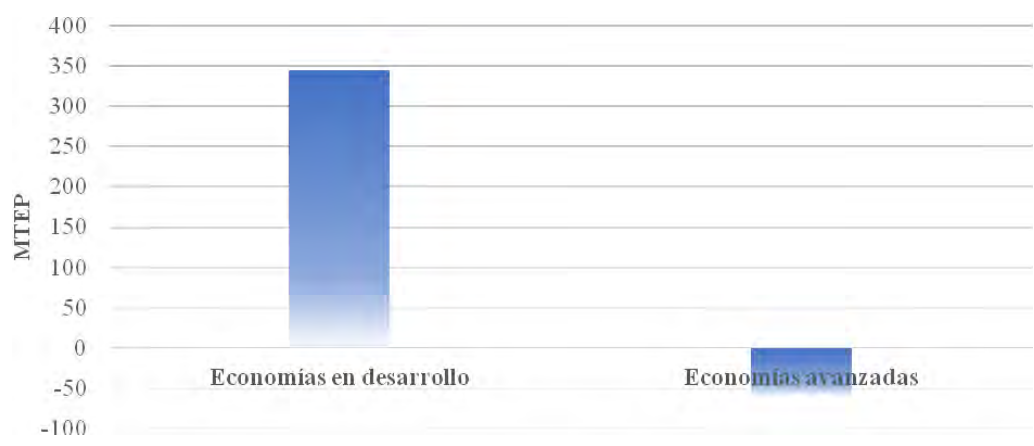

* Megatoneladas equivalentes de petróleo, MTOE por sus siglas en inglés. Fuente: elaboración propia con datos de iea World Energy Outlook, 2018.

El rechazo hacia la energía nuclear no necesariamente responde a intentar evitar cualquier riesgo de largo plazo que represente estas plantas, sino que es resultado del análisis económico sobre este tipo de proyectos. Ahora bien, esta tendencia antinuclear en economías desarrolladas se incrementa por la relativa holgura financiera que estos países tienen para migrar y reconfigurar sus modelos energéticos buscando la eficiencia monetaria a largo plazo. El Informe anual sobre el estado de la industria nuclear mundial (2019) especifica que:

El tiempo de construcción promedio de los reactores en todo el mundo fue de menos de 10 años, muy por encima de la estimación dada por la Asociación Nuclear Mundial (wna) de entre cinco y 8.5 años. El tiempo extra que las plantas nucleares tardan en construir tiene implicaciones importantes para los objetivos climáticos, ya que las plantas existentes alimentadas con combustibles fósiles continúan emitiendo $\mathrm{CO} 2$ mientras esperan su sustitución [...] la energía nuclear también es mucho más cara. El costo de generar energía solar varía de 36 dólares a 44 por megavatio hora (MWh), mientras que la eólica terrestre es de 
29 dólares a 56 por MWh. La energía nuclear cuesta entre 112 y 189 dólares (Roca, 2019).

En sentido puramente económico, el hecho de que crear plantas de energía nucleoeléctrica tarde muchos años y sea más costosa que otras tecnologías, orienta a estas naciones hacia la adopción de proyectos eólicos y solares, los cuales si bien son más ineficientes ${ }^{5}$ en la provisión de electricidad, son mucho más baratos y en general son mejor aceptados porque los daños ecológicos y humanos que registran son a menor escala. ${ }^{6}$

Para el caso específico de México y su sistema energético, se pueden diferenciar 13 tipos de tecnologías que generan electricidad en el país, los cuales varían notablemente en su tiempo de vida; éstos se muestran a continuación en la siguiente tabla.

Tabla 1. Tipos y costos de provisión de energía eléctrica en México

\begin{tabular}{cccc}
\hline Tipo de energía & $\begin{array}{c}\text { Tiempo de } \\
\text { vida en años }\end{array}$ & $\begin{array}{c}\text { Costo medio } \\
\text { de inversión } \\
\text { (dólares } / k W \\
\text { año) }\end{array}$ & $\begin{array}{c}\text { Costos fijos } y \\
\text { variables de } \\
\text { operación } y \\
\text { mantenimiento } \\
\text { promedio } \\
\text { (dólares } / k W \text { año) }\end{array}$ \\
\hline Hidroeléctrica & 60 & 1,931 & 24.4 \\
Nucleoeléctrica & $60^{*}$ & 3,988 & 103.5 \\
Carboeléctrica & 40 & 1,425 & 36.2 \\
\hline
\end{tabular}

5. Las tecnologías solar y eólica suelen tener una eficiencia productiva menor al $50 \%$ de su capacidad por la variación en las condiciones climáticas, de igual forma requieren una amplia extensión de territorio para generar la misma cantidad de electricidad que otras alternativas tecnológicas. Por otro lado, una planta nuclear tiene una eficiencia superior a $90 \%$ en el mejor de los casos y la cantidad de territorio que requiere es poca en comparación con los parques eólicos o solares. 6. No obstante, en la actualidad los combustibles fósiles siguen siendo la principal fuente de energía a nivel mundial según datos de la International EnergyAgency (IEA), que hasta noviembre de 2020 destacaban al gas natural con 235.8 teravatio-hora (TWh) producidos y al carbón con 165.6 TWh, frente a los I46.3 TWh de la energía nuclear y sólo 86.6 y 26. I TWh de la eólica y la solar, respectivamente (IEA, 202I). 


\begin{tabular}{cccc}
\hline Tipo de energía & $\begin{array}{c}\text { Tiempo de } \\
\text { vida en años }\end{array}$ & $\begin{array}{c}\text { Costo medio } \\
\text { de inversión } \\
\text { (dólares/ } k W \\
\text { año) }\end{array}$ & $\begin{array}{c}\text { Costos fijos } y \\
\text { variables de } \\
\text { operación } y \\
\text { mantenimiento } \\
\text { promedio }\end{array}$ \\
(dólares/ $k W$ año)
\end{tabular}

* Aplica únicamente a los reactores de generación III+, mientras que los usados en la CNLV son de tipo II con un tiempo de vida aproximado de 30-40 años.

** Este costo es variable, dependiendo del tipo instalado (5, 10, 50 y $100 \mathrm{Mw})$.

Fuente: elaboración propia con datos de Secretaría de Energía, 2018b.

En la tabla 1 se muestran las vastas diferencias de costos de inversión, operación y mantenimiento de las distintas tecnologías de generación de electricidad en el país. El hecho de que exista tal variedad es tanto por el enfoque operativo como por la naturaleza del servicio que van a ofrecer: empresas, hospitales, auxiliar, etcétera. De acuerdo con los datos de la tabla 1 se puede señalar que opciones como el turbogas parecen ser más viables en términos económicos por sus bajos costos; sin embargo, su eficiencia operativa es menor en comparación con otras opciones; además ésta se enfoca en proveer servicios auxiliares para cumplir con 
la alta demanda nacional, y de igual forma su nivel de contaminación es muy elevado.

Los costos económicos solamente representan una parte del panorama para analizar este tipo de proyectos, pero en este caso ayudarán a comprender los riesgos en diversos sentidos: económicos, sociales y ambientales de las centrales nucleoeléctricas. De acuerdo con Framatome - uno de los actores internacionales más importantes en la producción de reactores nucleares- el costo total de las centrales nucleoeléctricas se determina principalmente por los costos fijos de construcción: intereses del crédito y amortización del capital, así como el desmantelamiento de la planta (proceso que puede durar poco más de 65 años); dichos costos comprenden $70 \%$ del valor total de la inversión aproximadamente, y el restante se divide entre costos fijos y costos variables operativos (Thomas, 2010).

Uno de los argumentos más comunes que se utiliza a favor de la energía nuclear se refiere a que el tiempo de vida de los reactores es muy largo (en este caso el estimado es de 60 años, aunque el promedio para los que se emplean en la CNLV es de 30 a 40 años), proveyendo energía de forma constante (a diferencia de otras opciones como la solar y eólica, que tienen variaciones), por lo que podrían abaratar sus costos fijos en el largo plazo. El problema de este razonamiento es la valoración financiera en un entorno altamente competitivo de un bien como lo es la energía eléctrica, el cual además se encuentra afectado por las reglas operativas del mercado mexicano y controlado por tarifas con subsidios que distorsionan los precios de mercado. De manera que los costos del dinero para la inversión en este tipo de proyectos serán muy altos, pues existen pocas garantías de que no solamente debe ser rentable durante toda su operación, sino también para los fondos del desmantelamiento, lo que es un proceso de tan largo tiempo que tiene pocas opciones para financiarse de forma adecuada. 
Ahora bien, México ha planteado desde 2019 incrementar la capacidad nuclear del país instalando cuatro nuevos reactores, dos en la ya existente planta de Laguna Verde y dos más en la costa del Pacífico, según declaró ese año Héctor López Villareal, coordinador de Generación Termoeléctrica de CFE (Solís, 2019), mismos que no solamente son más eficientes que los que se disponen en la actualidad, sino también más seguros. La cuestión es que los reactores nucleares de nueva generación son extremadamente costosos, pues estimaciones independientes consideran plausible que este proyecto puede alcanzar los 10 mil millones de dólares (mmd), además la reconfiguración de los reactores viejos que pretendan superar su tiempo de vida normal ${ }^{7}$ pueden generar costos desde 3.5 hasta $6 \mathrm{mmd}$, de acuerdo con las estimaciones de World Nuclear Association (2019). Adicionalmente, el tiempo necesario para construcción e instalación es de 10 años aproximadamente.

En contraste, una planta solar o un parque eólico con un potencial parecido no solamente cuesta menos de una cuarta parte de esto, también es más barato en producción al comparar el costo nivelado (precio estándar de la electricidad) entre estas dos tecnologías, en la que el megawatt/ hora de la CNLV cuesta casi 100 dólares, mientras que el de la opción solar es de 22 dólares (PMCE, 2019). De igual forma, en este costo no se están considerando los gastos de almacenamiento del combustible nuclear ya gastado, mismo que debe quedar bajo resguardo durante décadas en áreas especiales.

Finalmente, respecto al proceso de desmantelamiento, se debe señalar que puede transcurrir en dos o tres etapas, dependiendo de las características de la planta. Este procedimiento requiere un alto nivel de vigilancia y control sobre la zona cuando se cierra la operación productiva, para evitar

7. En la reglamentación estadounidense un reactor de generación II como el de México podría extender su tiempo de vida entre 15 y 25 años. 
accidentes. Dado que requiere varias décadas completarlo, existen numerosos elementos de riesgo y falta de previsión que podrían afectar a la población y al ecosistema durante ese tiempo, haciéndolo una etapa especialmente peligrosa.

Los datos que se han mencionado hasta ahora, así como algunos otros no abordados por cuestión de espacio, ${ }^{8}$ demuestran que la energía nuclear actualmente presenta aspectos controversiales en términos económicos o de seguridad pública debido a sus posibles efectos en el ambiente y en la salud humana, pero para tener un panorama más completo de la situación se deben de considerar los aspectos sociales y ambientales, ya que de ellos depende la vida humana y la naturaleza. Por ello se revisan y analizan las protestas de la sociedad, especialmente las del grupo Madres Veracruzanas, por ser las que radican en ese estado.

\section{Madres Veracruzanas y su lucha contra la Central Nucleoeléctrica de Laguna Verde}

En 1986 empezó una serie de protestas contra el complejo nuclear CNLV, impulsada sobre todo por el accidente del 26 de abril de 1986 en Chernobil, unss, que dejó como resultado inmediato 31 personas muertas por el contacto

8. Se sugiere revisar el trabajo de Picquart, Zepeda y Carrasco (2016), en el cual se argumenta ampliamente sobre las desventajas de la energía nuclear en distintos ámbitos. Por su parte, Buncom (2018) recupera las dificultades para lidiar con los riesgos de los desechos nucleares y las dificultades para mitigar sus efectos socioambientales, superando estos problemas a los beneficios derivados de esta práctica. En el caso del reporte de la Conferencia Internacional Nuclear del Atlántico de 2017 en Brasil, si bien se menciona que los mayores problemas que enfrenta esta tecnología son la confianza de la gente debido al alcance de los posibles daños que podría provocar un accidente, lo cierto es que las ventajas que tiene la energía nuclear sobre otras formas de generación energética son mínimas y apenas justificables si se consideran los costos y tiempos de construcción. Otro trabajo interesante sobre el tema es el de Akyuz (2017), quien discute la importancia de la percepción pública del tema para el caso de Turquía, destacando que la población cercana a estos espacios está bien informada sobre el tema y suele mostrar preocupación sobre cuestiones relevantes que el Estado y la iniciativa privada no han comunicado de forma clara. 
directo con químicos radioactivos, además de 116,000 desplazadas y 600,000 expuestas a radiación que tuvieron como consecuencia serios problemas de salud. ${ }^{9}$ Las personas inconformes en Veracruz consideraron que la planta nuclear representaba una amenaza y una bomba de tiempo, por lo que comenzaron a organizarse en rechazo a este proyecto, en especial un grupo de 30 madres de familia originarias de Xalapa, quienes conformaron el 25 de febrero de 1987 el Grupo Antinuclear de Madres Veracruzanas, A. C., que tenía como objetivo frenar la construcción de la planta.

Este grupo representa un caso sin precedentes debido a que constituye una de las primeras luchas por la defensa socioambiental formulada por mujeres (profesionistas y amas de casa) de todas las edades, además de que algunas de sus integrantes han delegado su papel en hijas o nietas, por lo que es una resistencia que se ha transmitido de generación en generación. Las estrategias que han implementado tienen un gran valor simbólico, han empoderado a la mujer sin que fuera ése el objetivo principal y el grupo continúa vivo a 32 años de su fundación.

Margarita Juárez, su hija Mirna Alicia Benítez Juárez, Carolina Chacón y Adela Chacón Vega, ex integrantes de Madres Veracruzanas recordaron, durante una plática efectuada en Xalapa en 2016, que nadie se oponía a Laguna Verde hasta que ellas alzaron la voz. Margarita Juárez comenta que su inquietud al respecto surgió desde que se enteró de la construcción de la planta y se cuestionaba “¿qué puedes hacer tú por tú familia en caso de un accidente nuclear? Pues voy a ver en qué puedo ayudar" (entrevista personal, 2016).

Las mujeres participantes en el grupo concuerdan en que esta experiencia fue un aprendizaje para ellas que las

9. A principios de este siglo se estimó que la cifra de personas muertas como consecuencia de este evento fue de al menos 30 mil y que la afectación en forma de padecimientos o riesgos se extiende hasta 10 millones (Santamarta, 200I). 
enseñó a sensibilizarse ante las causas sociales y que en los años más álgidos de la lucha trataban de convencer a otras mujeres para que se unieran a su causa, e incluso lograron que algunas de un alto nivel socioeconómico lo hicieran, por lo que consiguieron hacer a un lado diferencias de cualquier índole por un objetivo común (entrevista personal, 2016). Sin embargo, convencer a las habitantes de los poblados cercanos a la planta de las afectaciones que ésta tendría, representó un problema porque "ellas lo veían como una fuente de trabajo y muchas no nos querían; hubo algunas mujeres que sí se atrevieron, pero no muchas, los núcleos de movilización fueron Xalapa, el puerto de Veracruz y Córdoba" (entrevista personal, 2016).

La labor del grupo no fue fácil debido a que se enfrentaron precisamente con gente simpatizante al proyecto y las divisiones fueron acrecentadas por los mismos gobiernos municipales que lo promovían como algo benéfico para la sociedad civil. Además, las integrantes pasaron por una estigmatización al ser señaladas como "revoltosas" por manifestarse abiertamente, pero al final "entendimos que era preferible ser señaladas, que no defender una causa justa" (entrevista personal, 2016). A pesar de ello, decidieron arriesgarse e implementaron acciones diversas: bloquear carreteras, marchar desde Xalapa hacia Laguna Verde, ir a programas de radio y televisión, dar pláticas en escuelas y contactar a grupos opositores a la energía nuclear en otros países, etc., pero su principal estrategia fue la realización de un plantón afuera del Palacio de Gobierno del estado en Veracruz cada sábado desde 1988. La idea era imitar a las "Madres de la Plaza de Mayo" de Buenos Aires, Argentina.

Pero algo que hizo a Madres Veracruzanas especial, fue la participación familiar; por ejemplo, Carolina y Adela Chacón Vega son hermanas gemelas; Margarita Juárez y Mirna Benítez son madre e hija, y Claudia Gutiérrez de Vivanco, su mamá y su hija Adriana actualmente conti- 
núan con el movimiento. $\mathrm{Al}$ respecto, Mirna recuerda que ella como joven inquieta constantemente participaba en manifestaciones, provocando el disgusto de su madre, quien comenzó a involucrarse en la lucha contra Laguna Verde y terminó pidiéndole apoyo para la organización de las protestas. Además, menciona la presencia de los hijos en las mismas y cómo su crecimiento se dio en este ambiente pero, paradójicamente, acompañado de reclamos por el tiempo que no pudieron compartir con ellas de otra manera (entrevista personal, 2016).

La labor de Madres Veracruzanas se ha visto obstaculizada también por las constantes intimidaciones de que han sido objeto sus integrantes desde la fundación del grupo. No obstante, a pesar de que esta agrupación rechaza un proyecto energético de gran calado e importancia para el país a nivel económico, lograron entrevistarse dos veces con el entonces presidente de la República, Carlos Salinas de Gortari, ello en gran medida gracias a las estrategias tan particulares que decidieron adoptar.

Una reunión con el presidente fue en el Hotel Xalapa y la otra por Catemaco, al sur del estado, nos dieron la oportunidad de hablar con él en el trayecto de un pueblo a otro [...] Nos subimos al camión presidencial, toda la comitiva de Salinas de Gortari iba sentada y nosotros como si fuéramos en un vagón del metro para allá y para acá, y luego cuando terminó de escucharnos nos bajaron en la noche a media carretera [...] su única respuesta fue que nuestro gobernador, que era Dante Delgado en ese entonces, era el mejor canal para externar nuestras preocupaciones; pero eso fue cuando nos recibió [...] las otras veces nos presentábamos en cualquier lugar, una vez inauguraron el Museo Interactivo de Xalapa, entonces era una ceremonia muy fastuosa y entramos muy propias con unas cartulinas escondidas debajo de la ropa, cuando estaba el momento preciso las sacamos y se las pusimos así enfrentito al hombre delante de todas las autoridades, estaba Zedillo, 
el gobernador Dante, Ricardo Rocha [...] nosotras nos pasamos muy propias, como invitadas (entrevista personal, 2016).

Otra acción pacífica, pero contundente fue entregarle una rosa a la esposa del gobernador del estado cada sábado al terminar el mitin, ésta iba acompañada de mensajes como "lo que quiero para mis hijos lo quiero para los suyos", hasta que "nos pidieron que por favor ya no mandáramos más rosas" (entrevista personal, 2016). Incluso, fue tanta la presión que ejercieron ante las instituciones gubernamentales, que se les ofreció un viaje a Chernobil con todos los gastos pagados para que vieran que "no pasaba nada", propuesta que rechazaron.

En este sentido, el movimiento de Madres Veracruzanas actuaba en concordancia con lo descrito por Touraine (2006), pues identifican a su opositor en la organización pública, pero es en la figura de la esposa del gobernador (aunque ésta no tenga poder político, pero sí influencia) que pueden apelar a su interlocutor, tanto por identidad como el enjeu (la vida propia y de sus conocidos), en una intervención institucional que refleja su acción en el riesgo que pueden sufrir todos los actores involucrados. La valoración instintiva del movimiento actúa debido a la lógica del funcionamiento social (familia-comunidad) y no desde el proceso de desarrollo (político), por lo que este tipo de presión puede ser mucho más efectiva en este escenario.

$\mathrm{Al}$ preguntarles sobre el logro más grande del grupo, Margarita Juárez afirma que fue "que las mujeres empezaron a participar y a tener conciencia” (entrevista personal, 2016). Adela Chacón refiere que además

[...] la lucha influyó para que se hiciera una revisión al plan de emergencia de Laguna Verde, porque en un inicio las indicaciones de qué hacer en caso de un accidente eran risibles y se llegaron a mejorar. Por ejemplo, decían que en caso de accidente te podías proteger de la 
radioactividad con un pañuelo o que se eliminaba bañándose.También se repartieron tabletas de yodo para contrarrestar la radiación (entrevista personal, 2016).

Se asume que la población está expuesta a un tipo de envenenamiento que requiere un medicamento especial de emergencia (tabletas de yodo), sin capacitar sobre el uso de éste y tampoco se informan las contraindicaciones que generan nuevos riesgos. Es decir, el desarrollo de un proyecto de este calado requiere completo y absoluto conocimiento sobre las implicaciones o efectos que puede generar, principalmente sobre las personas cercanas al mismo. Los testimonios comentados demuestran justo lo contrario y manifiestan una conducta irresponsable por parte del Estado que podría derivar en efectos irreparables para la humanidad y el ecosistema.

El grupo se debilitó después de 2010 debido al deterioro físico y emocional de las integrantes que llevaban ya más de 20 años participando. Actualmente Madres Veracruzanas sigue vigente gracias a Claudia Gutiérrez de Vivanco y su familia, que continúan manifestándose en la plaza principal de Xalapa cada sábado de 12 a 13 horas.

Maldonado recuerda así el debilitamiento de las protestas contra la planta nuclear:

La planta ya estaba instalada, pero seguían las manifestaciones [...] Y como no hubo respuesta del gobierno y la planta se echó a andar, la gente también se fue cansando. Fueron varias cosas. Porque el movimiento, en su parte fuerte, álgida, empezaron a cooptarlo, ofreciéndole puestos a ciertos dirigentes. Hubo un líder ganadero que después fue alcalde de Veracruz [...] Y así. Ésa es una parte, digamos que hasta "noble", porque hubo, no lo puedo comprobar, pero sí hubo comentarios de muchas gentes, que a muchas de las madres las empezaron a amenazar con quitarles el puesto de trabajo a sus familiares, para que le bajaran (Alpízar, 20II). 
Ahora, Laguna Verde vuelve a ser tema central desde que se anunció en 2017 un análisis sobre su posible ampliación. En 2018, la Comisión Federal de Electricidad (CFE) informó que se puede aumentar en 2.7 veces la producción energética. "Para cumplir el objetivo, se añadirían 2 mil 800 Mw a la capacidad instalada actual de la nucleoeléctrica que es de mil 608 mw" (Cruz, 2018). Recientemente el presidente Andrés Manuel López Obrador anunció: "solicité un informe, ya se me presentó. En ese informe no existe ningún riesgo en la planta [Laguna Verde] y tiene una certificación de un organismo internacional que se hace cargo de la seguridad" (Hernández, 2019). Por otro lado, la secretaria de Energía, Rocío Nahle confirmó la ampliación:

[...] tras escuchar las bondades de esta energía limpia del secretario estadounidense Rick Perry durante una visita en Houston, Estados Unidos, el cual está generando más electricidad mediante la fisión nuclear [...] Nahle agregó que las plantas nucleoeléctricas son nobles si se operan bien y con múltiples candados de seguridad (Solís, 2019a).

Esto es preocupante, porque grupos ecologistas han denunciado las malas condiciones en las que se encuentra la planta: grietas en un reactor, falta de simulacros para evaluar una posible catástrofe y malos caminos de acceso y de evacuación que impedirían que ésta se realizará de manera rápida. Además:

La central nuclear tiene problemas serios: ha permitido que algunos de sus trabajadores queden expuestos a dosis altas de radiación, ha tenido "paros automáticos" por problemas técnicos y no maneja adecuadamente sus residuos. Por estas razones, la Asociación Mundial de Operadores Nucleares le dio una calificación reprobatoria, pero los directivos de la planta ocultan y minimizan esta información (Godoy, 2019). 
Por otra parte, desde el año 2004 se ha reportado que el grupo Madres Veracruzanas observó en la zona que rodea a Laguna Verde un mayor nivel de incidencia de cáncer, malformaciones y otras enfermedades relacionadas con la radiación, debido, sobre todo, a la gran cantidad de incidentes (más de 50) que ha tenido la planta, como: derrames, malos manejos de desechos, entre otros (Azamar, 2019; Godoy, 2019; Viayra, 2004). Asimismo, también se mencionan enfermedades en la piel y malformaciones desde que se inauguró la planta, sin que hasta ahora se hayan hecho estudios detallados en los asentamientos cercanos a pesar de las múltiples peticiones al respecto.

En este sentido, a nivel local han surgido distintas denuncias por parte de trabajadores de la misma planta, así como de pobladores cercanos que buscan visibilizar el alcance de los daños de la energía nuclear. Este tipo de hechos han sonado con mayor fuerza en los últimos años. En 2011 familias de comunidades cercanas a Laguna Verde se manifestaron en el Congreso estatal para pedir que se investigaran 150 casos de cáncer en los municipios de Vega de Alatorre, Actopan y Emiliano Zapata, petición que no fue escuchada (Morales, 2011). Ese mismo año la presidenta municipal de Vega de Alatorre denunció al menos 108 casos de cuadriplejía, paraplejía, cáncer, problemas endocrinólogos y parálisis cerebral en personas de la localidad que vivían a menos de $60 \mathrm{~km}$ de la nucleoeléctrica y lamentó que "ninguno de los 28 municipios cercanos a Laguna Verde recibe información sobre los estudios de radiactividad que supuestamente realiza la Comisión Federal" (Méndez, 2011).

Por otra parte, Bernardo Salas Mar (académico de la UNAM) ha denunciado que desde hace años existe una fuga de cesio 137 en Laguna Verde, un químico radioactivo que contamina el agua y que puede incluso conducir a la muerte por envenenamiento (Garza, 2019). Asimismo, Ana Rodríguez Valladares, ex trabajadora de la central nuclear, demandó 
a la CFE "por exponerla a altas dosis de radiación, lo que ha mermado su salud [...] durante la recarga de combustible de uno de los reactores de la central, la radiación emitida por tuberías y válvulas afectó a cinco trabajadores [...] la mujer recibió una alta dosis de cobalto y los peritajes de salud realizados así lo comprueban" (Godoy, 2020).

De este modo resultan preocupantes las amenazas a la salud humana y la poca regulación con que opera Laguna Verde. En este sentido la Comisión Nacional de Seguridad Nuclear y Salvaguardias (CNSNS) realizó el Informe de rendición de cuentas de conclusión de la administración 2012-2018 (CNSNS, 2018) sobre la CNLV, en el que se detalla que, si bien existe una gran cantidad de mecanismos y controles de seguridad que se han realizado de manera adecuada a pesar de las carencias económicas y de otros recursos materiales, lo cierto es que hay varias cuestiones que parecen preocupantes. Por ejemplo: a) que no se cumple el estándar mínimo de cantidad de inspecciones a las instalaciones de seguridad; b) se carece de asesoría externa y estudios de análisis especializados en materia nuclear; c) poca o nula vinculación con proyectos de investigación científica nacional; d) existen múltiples equipos en estado de obsolescencia que ponen en riesgo al personal; e) así como la fiabilidad sobre los informes de seguridad; f) incapacidad de cumplir con las medidas de seguridad mínimas para operar adecuadamente la planta debido a los techos presupuestales, entre otras cuestiones.

Muchos de estos problemas derivan de la reducción presupuestaria a esta planta nuclear desde el año 2016, limitando su capacidad económica hasta $40 \%$, lo que se suma al déficit que ya acumulaba, pues el presupuesto asignado para su operación era menor al que requería realmente para llevar a cabo su actividad de forma segura, lo que implica efectos perversos en la naturaleza política y económica que impulsa este proyecto nuclear, pues en caso de que no se maneje adecuadamente la CNLV provocaría altos niveles de 
contaminación, por lo que se le imponen castigos presupuestarios, exigiendo el mismo nivel de productividad.

Asimismo, representa un verdadero retroceso en materia ambiental el hecho de que México apueste por incrementar su producción de energía nuclear cuando países europeos han decidido dejar de usarla debido a las afectaciones que ésta puede tener. Mientras otras naciones están implementando acciones para abandonar este tipo energía, nuestro país está pensando en aumentarla. Por ejemplo, Francia, el segundo país en explotar este tipo de energía, con 58 centrales nucleares en activo, precisó que tiene el objetivo de reducir a la mitad su producción en el año 2025 (Redacción, 2018).

Es notorio que en este proceso se cultivan conflictos y rechazo de la población tanto a la estructura política - al observarla como cómplice del proyecto destructivo- como al sector privado (contratista en la construcción, mantenimiento y actividades diversas), siendo partícipe del daño y de los problemas que se generan en la región. Los conflictos socioambientales emanados de estos proyectos se afincan en la interpretación errónea del interés que existe entre la población y el Estado, pues para este último su propia existencia se basa en la narrativa de crecimiento económico y desarrollo occidental (encarnado en crecimiento demográfico y productivo), mientras que para la comunidad, al ser heterogénea y estar fragmentada en grandes matices de inequidad y marginación, hay una absoluta desconexión con el modelo de desarrollo que desconoce la subalternidad de existencias que dan lugar a la riqueza del lugar y del territorio, por lo que se fomentan, impulsan y potencian tensiones de territorialidades que se resuelven mediante el conflicto.

\section{Conclusiones}

En este trabajo se analiza el efecto social que provoca el proyecto CNLV en Veracruz al generar una situación de 
organización y resistencia que se ha prolongado por 30 años por medio del colectivo de Madres Veracruzanas; asimismo, se revisa si es que existe viabilidad del planteamiento del Gobierno mexicano por el interés de la ampliación que se tiene sobre la energía nuclear en nuestro país. De acuerdo con los datos planteados y el análisis elaborado en este trabajo, la CNLV presenta algunas ventajas, como la uniformidad en la creación de energía, así como la eficiencia productiva que es superior a otras opciones como la solar o la eólica; sin embargo, los elementos negativos que caracterizan a esta planta nucleoeléctrica sobrepasan por mucho los beneficios. De acuerdo con los datos disponibles:

1. No solamente es más caro producir energía nuclear en el país, también los costos de actualización son extraordinariamente altos en comparación con casi cualquier otra fuente productiva, ya que presentan desventajas económicas de financiamiento y carecen de garantías suficientes para ser respaldadas en condiciones adecuadas para México.

2. Por si fuera poco, los riesgos de largo plazo que implica el desmantelamiento de la planta significan una deuda ecológica y social que pueden representar un gran peligro a futuro, lo cual, conociendo el historial de faltas que tiene el Estado, son un sinónimo de amenaza latente.

3. Por otro lado, desde el aspecto social es importante resaltar la conflictividad socioambiental generada en la entidad veracruzana como resultado del proyecto de la CNLV, en el que se observa una resistencia a éste a partir de una acción colectiva. Asimismo, este caso es representativo por las condiciones en las que se ha ido desarrollando, pues se ha convertido en un referente sobre los procesos de la defensa socioambiental por sus propias características de lucha. 
4. La contradicción y enfrentamiento entre estas formas de realización social, económica y ecológica da origen a las tensiones territoriales entre los actores involucrados. La exclusión de los participantes que comúnmente no son reconocidos (como las mujeres) se manifiesta como una fuerza opuesta y contraria a la valoración puramente económica de la agenda política de explotación territorial que se pretende llevar a cabo en el estado de Veracruz.

5. En la misma naturaleza reflexiva se debe destacar la importante y constante actividad de resistencia de las mujeres en el movimiento contra la cNLV, pues en su concepción de vida se reconoce la amenaza de este proyecto productivo hacia su tejido social y comunitario; asimismo, se distingue que es en el Estado donde reside el proceso de explotación como garante de la normativa que permite el accionar de esta actividad, que aunque no pueda ser resuelto por quienes comenzaron el movimiento, sí será heredado a sus hijas y conocidas de manera que pueda seguirse la resistencia en el tiempo, lo que representa un rechazo al modelo de explotación y aprovechamiento de la naturaleza, como también una lucha por parte de las mujeres.

Es en esta perspectiva que el movimiento de Madres Veracruzanas se contrapone a la narrativa preponderante del Estado al enfrentarla y mantenerse constante en su resistencia a pesar de ser evidentemente superadas por los medios de los que dispone el Estado, por lo que existe un proceso de fortalecimiento del colectivo que incluso ha motivado a los gobiernos local y federal a responder a su demanda social. El hecho de que se otorgara dicha atención al colectivo se debe al largo y constante proceso de identificación de la causa (la amenaza nuclear) y de los afectados 
(la población), lo que ha sido un proceso tenaz, coincidiendo en la narrativa de Touraine y Porto.

Sin duda hace falta analizar más a fondo las problemáticas ambientales que la CNLV genera en este sentido, pero la intención de este trabajo es mostrar un breve diagnóstico sobre la viabilidad de la planta y el movimiento socioambiental antinuclear más emblemático en México que ha sido encabezado por mujeres.

Por otro lado, recientemente fue aprobado el Decreto por el que se reforman y adicionan diversas disposiciones de la Ley de la Industria Eléctrica, enviado por el presidente Andrés Manuel López Obrador al Legislativo, el cual tiene como objetivo dar prioridad a la CFE para abastecer el Sistema Eléctrico Nacional (SEN), relegando a la industria privada. Por lo tanto, será necesario observar la evolución del sector eléctrico y qué estrategias adopta el Estado en su papel de productor y distribuidor de energía, pues en primera instancia se espera un freno en los proyectos solares y eólicos, pero la nuclear se mantiene aún en la incertidumbre (Centro de Investigación en Política Pública, 2021). Sea como sea, los conflictos socioambientales parecen mantener una tendencia a la alta, lo que debe vigilarse muy de cerca ya que impacta directamente en toda la sociedad y el ecosistema, y los principales afectados siempre son los más vulnerables.

Por último, si bien la mayor preocupación del Estado mexicano no ha sido la parte ambiental —más bien se ha mantenido una perspectiva depredadora sin muchas diferencias hasta la actualidad-, lo cierto es que existen esfuerzos puntuales desde algunas instancias del nuevo gobierno para fortalecer la protección socioambiental. Sin embargo, aún es pronto para realizar un análisis al respecto, por lo que se debe mantener una revisión constante de estas iniciativas, en las que el bienestar socioambiental debe ser la base indiscutible. 
Bibliografía

Akyuz, Enes. (2017). Advantages and Disadvantages of Nuclear Energy in Turkey: Public Perception. Eurasian Journal of Environmental Research, I (I): I I I .

Albisu,Javier. (2020,6 de octubre). El urgente dilema nuclear de Bélgica. La Vanguardia. Recuperado de: https://www. lavanguardia.com/politica/2020 I006/48388820 I376/ el-urgente-dilema-nuclear-de-belgica.html

Alpízar, Javier. (201 I, 3I de marzo). Laguna Verde fue impuesta. Zapateando. Recuperado de: https://zapateando.wordpress.com/20 I l/03/3 I/laguna-verde-fueimpuesta/

Azamar, Aleida. (2019, II de octubre). La energía nuclear en México, una de las más inseguras del mundo. El Universal.Recuperado de:https://www.eluniversal.com.mx/ opinion/aleida-azamar/la-energia-nuclear-en-mexicouna-de-las-mas-inseguras-del-mundo

BP plc. (2019). BP Statistical Review of World Energy. BP plc. Recuperado de: https:/www.bp.com/content/dam/bp/ business-sites/en/global/corporate/pdfs/energy-economics/statistical-review/bp-stats-review-20 I 9-fullreport.pdf

Buncom, Frank. (20I8). Nuclear Waste Storage: Why We Should Avoid Nuclear Energy. Introduction to Nuclear Energy. Reports. Stanford University.

Celis, Darío. (2021, 5 de marzo). El contrato ventajoso, ahora, de GE en Laguna Verde. El Financiero. Recuperado de: https://www.elfinanciero.com.mx/opinion/dario-celis/ el-contrato-ventajoso-ahora-de-ge-en-laguna-verde

Centro de Investigación en Política Pública [en línea].(202I). Es un retroceso para México la iniciativa de reforma a la Ley de la Industria Eléctrica. Recuperado de: https:// imco.org.mx/es-un-retroceso-para-mexico-la-iniciativa-a-la-ley-de-la-industria-electrica/

Comisión Nacional de Seguridad Nuclear y Salvaguardias (CNSNS). (20I8). Informe de rendición de cuentas de con- 
clusión de la administración 20 / 2-20 / 8. México: Comisión Nacional de Seguridad Nuclear y Salvaguardias. Recuperado de: https://www.gob.mx/cms/uploads/attachment/ file/4I572I/cnsns_informe_consolidado_rc20I2-20I8. pdf

Cruz, Noé. (2018, 22 de junio).Analizan ampliar generación de energía nucleoeléctrica. El Universal. Recuperado de https://www.eluniversal.com.mx/cartera/analizan-ampliar-generacion-de-energia-nucleoelectrica

Da Mata, Jônatas F. C., Mesquita,Amir Z., y Neto, Rieder O. (2017). Comparison of the performance, advantages and disadvantages of nuclear power generation compared to other clean sources of electricity. International Nuclear Atlantic Conference (INAC).

El Financiero. (2019, 18 de septiembre). Incrementar la energía nucleoeléctrica que se produce en México, plantea Rocío Nahle. El Financiero. Recuperado de: https://www.elfinanciero.com.mx/economia/apostar-mas-a-la-energia-nucleoelectrica-plantea-rocio-nahle

Excélsior. (2018,5 de septiembre). Francia anuncia reducción nuclear para el año 2035. Excélsior. Recuperado de: https://www.excelsior.com.mx/global/francia-anuncia-reduccion-nuclear-para-el-ano-2035/I263I 03

Garza, Plácido. (2019, II de octubre). Experto advierte contaminación radioactiva en Laguna Verde. SDP Noticias. Recuperado de:https://www.sdpnoticias.com/columnas/ placido-garza-laguna-verde-problemas-radioactivos.html Gebara, Ivone. (2000). Intuiciones ecofeministas. Ensayo para repensar el conocimiento y la religión. Madrid, España: Trotta.

Godoy, Emilio. (2019,2I de marzo). Laguna Verde una bomba de tiempo envuelta en mentiras. Proceso. Recuperado de:https://www.proceso.com.mx/576203/laguna-verde-una-bomba-de-tiempo-envuelta-en-mentiras 
Bibliografía
. (2020, 3 de enero). Juez ordena a la CFE atención médica a ex trabajadora de Laguna Verde por contaminación radioactiva. Proceso. Recuperado de: https://www. proceso.com.mx/nacional/2020/I/3/juez-ordena-la-cfe-atencion-medica-extrabajadora-de-laguna-verde-por-contaminacion-radioactiva-236605.html

González, Fabiola. (2019, I 4 de marzo).Veracruz, foco rojo por alta incidencia de conflictos ecológicos. Diario de Xalapa. Recuperado de: https://www.diariodexalapa. com.mx/local/veracruz-foco-rojo-por-alta-incidencia-de-conflictos-ecologicos-3 I84404.html

Hernández, Javier. (2019, I I de febrero). No existe ningún riesgo en planta de Laguna Verde:AMLO. El Dictamen. Recuperado de: https://www.eldictamen.mx/2019/02/ veracruz/estatal/no-existe-ningun-riesgo-en-planta-de-laguna-verde-amlo/

International Energy Agency (IEA). (20I8). World Energy Outlook 2018. IEA. Recuperado de: https://www.iea.org/ reports/world-energy-outlook-2018

- (202I). Monthly electricity statistics. IEA. Recuperado de: https://www.iea.org/reports/monthly-electricity-statistics

Kuri Pineda, Edith. (20I6). El carácter multidimensional de la acción colectiva y los movimientos sociales: Una problematización teórica. Secuencia, núm. 95, pp. 188214. Disponible en:http://secuencia.mora.edu.mx/index. $\mathrm{php} /$ Secuencia/article/view/ 382

LaVanguardia. (2019, 20 de diciembre). Suiza cierra su primera central nuclear y avanza hacia el fin de esa energía. LaVanguardia. Recuperado de: https://www.lavanguardia. com/vida/20I9/220/472369/44260/suiza-cierra-suprimera-central-nuclear-y-avanza-hacia-el-fin-de-esaenergia.html

—. (2020, 2 de junio). Alemania quiere ser verde. La Vanguardia. Recuperado de: https://www.lavanguardia. 
com/vida/junior-report/20200602/48 I 547049 I38/aleBibliografía mania-nuclear-energias-renovables.html

Méndez, Ernesto. (201 I, I 8 de abril). Registran 108 enfermos en un municipio aledaño a Laguna Verde. Excelsior. Recuperado de: https://www.excelsior.com.mx/node/730677

Morales,Andrés. (201 I, 9 de noviembre).Atribuyen a Laguna Verde casos de cáncer. La Jornada. Recuperado de: https:// www.jornada.com.mx/20I I/I I/09/estados/040n7est

Paz Salinas, María Fernanda. (20/4). Conflictos socioambientales en México: ¿Qué está en disputa? En M. Fernanda Paz y N. Risdell (coords.), Conflictos, conflictividades y movilizaciones socioambientales en México: Problemas comunes y lecturas diversas (Pp. I3-58). México:UNAM/CRIM/Porrúa. Picquart, Michele, Zepeda, Marco Antonio, y Carrasco, Izayana. (2016). Energía nuclear, contaminación radioactiva y sus efectos en la salud. México: Universidad Autónoma Metropolitana. Recuperado de: https://www.casadelibrosabiertos.uam.mx/contenido/contenido/Libroelectronico/Energia-nuclear.pdf

Plataforma México, Clima y Energía (PMCE). (2019). Costo de generación de energía. ¿Cuánto cuesta la electricidad? De los costos del sistema a los costos de generación. Ponencia presentada en el Taller de Medios: Entendiendo el sector eléctrico, México, I 3 de junio. Recuperado de: https:// www.pmce.mx/eventos-anteriores

Porto Gonçalves, Carlos Walter. (200I). Geografias: Movimientos sociales, nuevas territorialidades y sustentabilidad. México: Siglo XXI Editores.

Roca, José. (2019, 25 de septiembre). La nueva energía nuclear es demasiado lenta y excesivamente cara para salvar el clima. El Periódico de la Energía. Recuperado de: https://elperiodicodelaenergia.com/la-nueva-energia-nuclear-es-demasiado-lenta-y-excesivamente-cara-para-salvar-el-clima/ 
Bibliografía
Rodríguez, Israel. (2019, 19 de septiembre). Propone Nahle ampliar la capacidad de la nucleoeléctrica de Laguna Verde. La Jornada. Recuperado de: https://www.jornada. com.mx/2019/09/19/economia/030n3eco

Santamarta Flores, J.(200I). El cierre de Chernobil no acaba con la pesadilla nuclear. Papeles del Este. Transiciones Poscomunistas, núm. 2, pp. I0-13. Recuperado de: https:// www.nodo50.org/worldwatch/ww/pdf/chernobil.pdf

Secretaría de Energía (Sener). (20l8a). Reporte de avance de energías limpias. Primer semestre 2018. México:Sener. Recuperado de:https://www.gob.mx/cms/uploads/attachment/file/4 I839I/rael_primer_semestre_20I8.pdf

_ (2018b). Prodesen. Programa de Desarrollo del Sistema Eléctrico Nacional. México:Sener. Recuperado de: https:// www.gob.mx/cms/uploads/attachment/file/33 I 770/ prodesen-2018-2032-definitiva.pdf

Solís,Arturo. (2019a, 18 de septiembre). Gobierno de AMLO contempla generar más electricidad con energía nuclear. Forbes. Recuperado de: https://www.forbes.com.mx/ gobierno-de-amlo-contempla-generar-mas-electricidad-con-energia-nuclear/

- (2019b, 10 de septiembre). CFE estudia instalar 4 reactores nucleares más en México. Forbes. Recuperado de: https://www.forbes.com.mx/cfe-estudia-instalar-4-reactores-nucleares-mas-en-mexico/

Thomas, Steve. (2010). Los costos de la energía nuclear: Una actualización. (Volumen 3). Chile: Fundación Heinrich Böll Stiftung. Recuperado de: https://cl.boell.org/sites/ default/files/costos_de_la_energia_nuclear.pdf

Touraine, Alain. (2006). Los movimientos sociales. Revista Colombiana de Sociología núm. 27, pp. 255-278. Recuperado de: https://revistas.unal.edu.co/index.php/recs/ article/view/7982/8626

Varios, comunicación personal.(2016). Entrevistas personales "Madres Veracruzanas". 
Viayra Ramírez, Mariana. (2004, 2 I de septiembre). DenunBibliografía cian más de 80 casos de cáncer y malformaciones en Laguna Verde. La Crónica. Recuperado de: http://www. cronica.com.mx/notas/2004//44859.html

World Nuclear Association. (2019). Nuclear Power in the USA [en línea].World Nuclear Association. Recuperado de: https://www.world-nuclear.org/information-library/ country-profiles/countries-t-z/usa-nuclear-power.aspx 